\title{
Low-Noise Low-Mass Front End Electronics for Low-Background Physics Experiments Using Germanium Detectors
}

\author{
Paul Barton, Paul Luke, Mark Amman, Yuen-Dat Chan, Jason Detwiler, James Loach, Ryan Martin, Alan Poon, \\ Craig Tindall, and Kai Vetter
}

\begin{abstract}
A low-noise resistive-feedback front-end electronics assembly has been developed for use with p-type point contact (PPC) Ge detectors in low background experiments. The front end was designed to have a low mass and potentially low radioactivity. It is fabricated on a fused silica substrate, and consists of a low-noise JFET, a feedback resistor formed from an amorphous Ge thin film, and feedback capacitor based on the stray capacitance between circuit traces. The substrate provides the appropriate thermal impedance to allow the FET to operate at the optimal temperature from self-heating when one side of the substrate is held at liquid nitrogen temperature. A noise level of $85 \mathrm{eV}$ FWHM at 20 us peaking time has been observed in combination with a small PPC detector, the 1/f contribution being as low as $30 \mathrm{eV}$ for the front end alone. This approach of employing ultra-low-mass and low-noise front-end electronics in combination with larger-size PPC detectors can be an enabling technology towards the observation of particles and processes such as neutrino-less double-beta, coherent neutrino scattering or cold dark matter.
\end{abstract}

\section{INTRODUCTION}

A number of low-background experiments using HPGe detectors are being planned or constructed, including searches for neutrino-less double-beta $(0 v \beta \beta)$ decay in ${ }^{76} \mathrm{Ge}$ (MAJORANA) [1], cold-dark matter search (CDMS) [2], and coherent neutrino nuclear scattering (CNNS) [3]. Ge-based ptype point-contact (PPC) detectors are well suited for such experiments since they are characterized by several properties necessary to measure the weak signals of interest $(\sim 100 \mathrm{eV})$ while minimizing the background radiation. Operating deep underground and selecting radio-pure and low-mass materials is essential to the observation of rare physical processes.

Traditional large-volume Ge detectors consist of a coaxial geometry with a capacitance on the order of tens of $\mathrm{pF}$. In order to reduce the intrinsic electronic readout noise, detectors of lower capacitance are desired. Point-contact detectors consist of cylindrical high-purity Ge crystals with one contact reduced to a small point $(\sim 1 \mathrm{~mm})$, thus achieving a fulldepletion capacitance of $\sim 1 \mathrm{pF}$ and significantly reducing the equivalent noise charge. The very pronounced weighting field close to the point contact also enables excellent discrimination

Manuscript received November 22, 2011. This work was supported in part by $[\mathrm{x}]$.

P. Barton, P. Luke, M. Amman, Y.D. Chan, J. Detwiler, J. Loach, R. Martin, A. Poon, C. Tindall, and K. Vetter are with the Lawrence Berkeley National Laboratory, Berkeley, CA 94702 USA (telephone: 510-486-6644, email: pjbarton@lbl.gov). of single- and multi-site events. The first point-contact detector was fabricated from n-type Ge, which showed significant spectral degradation due to electron trapping [4]. P-type point contact detectors have recently been produced that exhibit excellent spectral performance because their signals arise from hole collection and therefore not sensitive to electron trapping [5].

In order to further reduce the noise of the detector while at the same time minimizing the radioactive background, a lownoise low-mass front end (LMFE) resistive-feedback electronics assembly has been developed, depicted in Figure 1. The LMFE consists of an input JFET and feedback resistor and capacitor, which can be mounted close to the cold detector to both match the low PPC capacitance and minimize stray input capacitance. This paper describes the LMFE concept and the material selection, as well as its low energy performance in combination with several PPC detectors.

\section{FABRICATION}

The selection of materials for the LMFE must meet several intersecting guidelines. Their radioactive emissions must be as low as possible, which also dictates that there be very little mass and volume. Candidate materials must be vacuum compatible (i.e. low outgassing) and function predictably at or near $80 \mathrm{~K}$. The materials should be available reliably and in quantity to maintain sufficient quality control, and the method of fabrication and assembly should be repeatable.
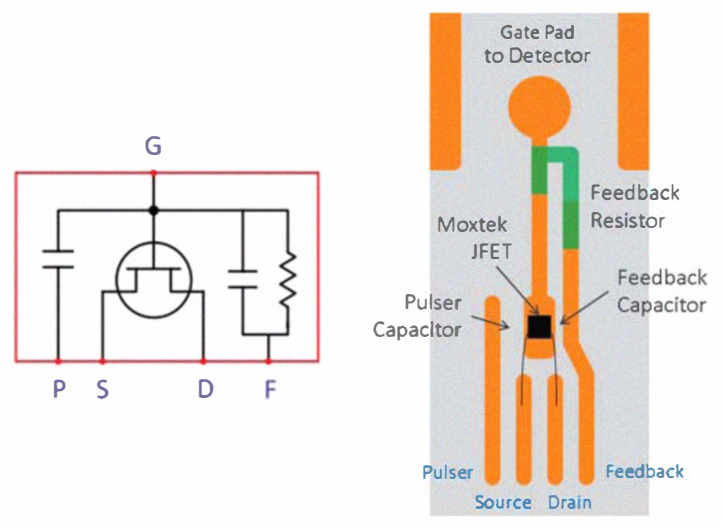

Fig. 1. Low-Mass Front End (LMFE) schematic and physical layout $(7 \times$ $16 \times 0.25 \mathrm{~mm}^{3}$ ) indicating locations of feedback resistor and capacitor, pulser capacitor and JFET. Traces in upper corners are clamped at $80 \mathrm{~K}$. 
The LMFE substrate is selected from radioactivity-assayed lots of DOW Corning HPFS ${ }^{\circledR} 7980$ fused silica, an amorphous silicon dioxide material with a very low coefficient of thermal expansion $(0.5 \mathrm{ppm} / \mathrm{K})$. It has a relatively low thermal conductivity suitable for thermal control of the JFET, and has low dielectric loss for minimizing $1 / \mathrm{f}$ noise. This material is also ideal for photolithographic processing.

Three-terminal JFETs (MX-11 or MX-11rc) from MOXTEK [6] are obtained as bare die $\left(0.9 \times 0.9 \times 0.51 \mathrm{~mm}^{3}\right)$, epoxied and then wirebonded to $\mathrm{Au}$ traces on the silica substrate. The MX-11 rc was originally designed for $\mathrm{Si}(\mathrm{Li})$, SiPIN and SDD detector readout, and its unique feature is an extremely low input capacitance (gate to source) of $0.7 \mathrm{pF}$. The transconductance $g_{m}$ is $\sim 8 \mathrm{mS}$ at $170 \mathrm{~K}$. A typical noise voltage of $1.6 \mathrm{nV} / \sqrt{\mathrm{Hz}}$ is stated at frequencies as low as 10 $\mathrm{kHz}$ (also at $170 \mathrm{~K}$ ).

The choice of JFET as front-end transistor necessitates an optimal operating temperature between $100 \mathrm{~K}$ and $200 \mathrm{~K}$. Due to the constant flow of current through the JFET, Joule heating will naturally raise the JFET's temperature from its 80 $\mathrm{K}$ liquid nitrogen temperature surroundings. The degree of heating and the geometry of the substrate must be chosen carefully to achieve temperatures that optimize the JFET leakage current, transconductance and noise properties.

The silver epoxy which bonds JFET to Au trace is a Henkel Hysol® TRA-DUCT 2902 Resin, selected for its low vacuum outgassing and temperature cycling stability. Radioactive assay has shown this epoxy to have suitable radiopurity. Care must be taken during application to avoid excess noise from poor mixing or curing.

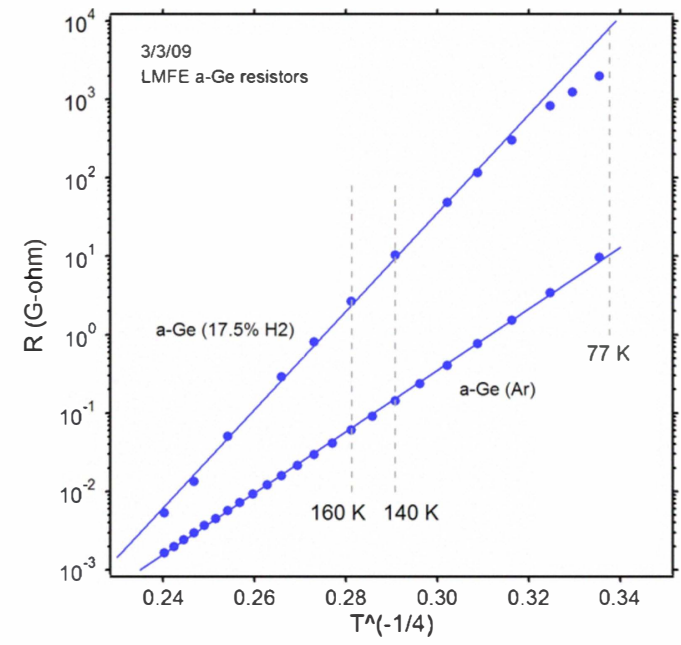

Fig. 2. Resistance in gigaohms of sputtered amorphous Ge thin films on a silica substrate, indicating strong variance with temperature. The inclusion of hydrogen in the Ar sputtering gas leads to a significantly higher resistivity.

The thin film feedback resistor is deposited from a highpurity Ge target sputtered in an $\mathrm{Ar} / \mathrm{H}_{2}(17.5 \%)$ atmosphere, and is chosen for its high resistivity and low $1 / \mathrm{f}$ noise. Placement of this resistor on the thermally graded substrate is critical, because the $\mathrm{Ge}$ resistance varies as $\exp \left(\mathrm{T}^{-1 / 4}\right)$. A resistance of 1-100 G $\Omega$ is readily achieved (Fig. 2) with variation of dimension and/or temperature. In the current design, the amorphous Ge resistor is placed near the cold end of the board. This maintains a more stable temperature for the resistor and the lower temperature reduces its Johnson noise.

Circuit traces of $\mathrm{Au}$ are required for low resistance and suitable wirebonding. Aluminum is difficult to obtain with sufficient radio-purity. Since $\mathrm{Au}$ does not adhere well to smooth glass, a thin adhesion layer such as $\mathrm{Cr}$ or $\mathrm{Ti}$ is sputtered prior to the Au. Standard photolithographic etching techniques are employed to pattern the relatively gross features $(0.25$ to $1 \mathrm{~mm})$. The proximity of traces is utilized to form both feedback and pulse-injection capacitors on the order of tens of fF.

Table 1. Preliminary radioactivity assay on LMFE components for the MAJORANA Collaboration [4].

\begin{tabular}{|c|c|c|c|c|c|c|}
\hline \multirow[t]{2}{*}{ Component } & \multirow[t]{2}{*}{ Material } & \multicolumn{2}{|c|}{ Purity $(g / g)$} & \multicolumn{2}{|c|}{ (ounts/ROI/t/y } & \multirow[t]{2}{*}{ Ref. } \\
\hline & & $23 \cdot 2 \cdot \mathrm{Ih}$ & 230 & ${ }^{232} \mathrm{Th}$ & ${ }^{2334} \mathrm{U}$ & \\
\hline Substratc & Fused silica & $101 \times 10^{-12}$ & $284 \times 10^{-12}$ & 0.0259 & 0.0616 & MJ ICP-MS \\
\hline Traces & $\mathrm{Au}$ & $47(1) \times 10^{9}$ & $2.0(0.3) \times 10^{9}$ & 0.0421 & 0.0015 & MJ ICP-MS \\
\hline Traces & $\mathrm{Ti}$ & $<400 \times 10^{12}$ & $<100 \times 10^{12}$ & $\sim 0$ & $\sim \mathbf{o}$ & MJ ICP-MS \\
\hline FET & FET die & $<2 \times 10^{-9}$ & $<141 \times 10^{-12}$ & $<0.0107$ & $<0.0006$ & MJ ICP.MS \\
\hline Bonding wire & Al & $91(2) \times 10^{-! 1}$ & $9.0(0.4) \times 10^{-12}$ & 0.0004 & $\sim 0$ & MJ ICP.MS \\
\hline Fpoxy & Silver epoxy & $<70 \times 10^{4}$ & $\therefore 10 \times 10^{\prime \prime}$ & $\therefore 0.0685$ & $\therefore 0.0082$ & M] ganma \\
\hline Tutal & & & & $<0.1476$ & $\checkmark 0.0720$ & \\
\hline
\end{tabular}

LMFE material selection must take into account the radioactive emission from the constituent components. Because the LMFE must be situated in close proximity to the detector in order to minimize stray capacitance, the materials involved must be relatively radio-pure. Materials have been assayed by inductively coupled plasma mass spectrometry (ICP-MS) or by direct gamma counting with HPGe detectors (Table 1). The fused silica is reasonably low in radioactivity due to its use in the laser window industry where optical purity is paramount. The a-Ge resistor is fabricated from high-purity Ge which is shown to be radio-pure. The JFET die is outside our control, but since it was fabricated from pure silicon substrate under clean processes, its radio-purity is sufficiently good. The total predicted radioactivity is currently $715 \mathrm{nBq} / \mathrm{LMFE}$ (from Th and U).

\section{PERFORMANCE}

The signals from the source, drain, and feedback of the LMFE are connected via a long parylene-coated multiconductor cable to a low noise discrete preamplifier of traditional design [7]. The LMFE is mounted in a tension-fit copper clip which holds one end of the board near LN temperature. Electrical connection to the detector is established via a 10-20 mm long copper pin (Fig. 3). The LMFE mounting clip then flexes to establish adequate pressure between the detector, pin, and the LMFE board. Care must be taken to adjust this tension as too little force may not produce a good electrical contact and may cause vibrations and microphonic noise, while too great a force can crack the thin silica substrate or damage the detector contact. Consideration must also be given to the varying rates of thermal contraction for various materials and assembly geometries. 


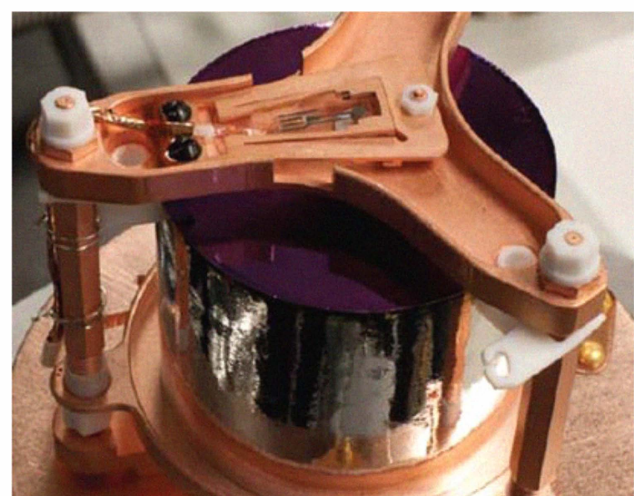

Fig. 3. MAJORANA PPC detector fabricated at LBNL, mounted in connection to a LMFE readout board.

A stable pulser is used to inject a fixed amount of charge through the detector into the LMFE. A radiation check source is then used to relate the amount of pulser-injected charge to a known energy deposition. The baseline electronic noise (Fig. 4) is then measured on a calibrated true RMS voltmeter (Boonton 93A) as a function of shaping time. Alternately, the noise can be determined by measuring the spectral width of the pulser peak using a multichannel analyzer. The components (series, parallel, $1 / f$ ) of the equivalent noise charge (ENC) curve obey linear relationships when the $\mathrm{ENC}^{2}$ is related to the FWHM of the shaping time in a log-log plot.

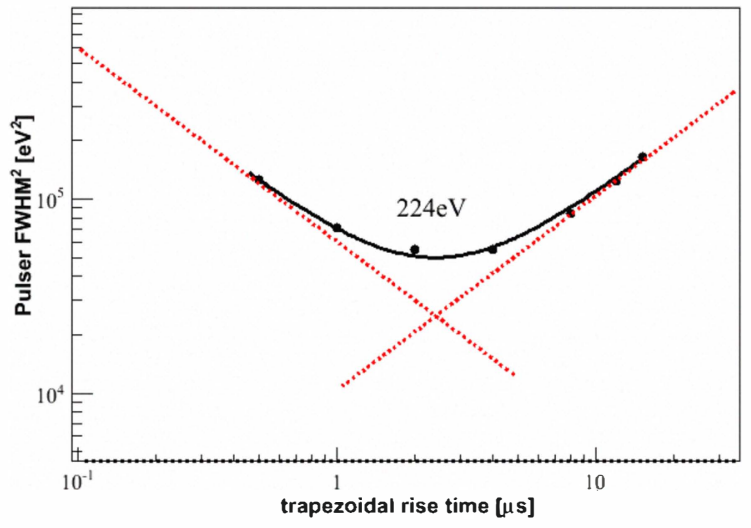

Fig. 4. Equivalent noise charge (squared) as a function of the trapezoidal shaper rise time for LBNL PPC detector from Fig. 3, indicating a minimum resolution of $224 \mathrm{eV}$.

The ENC in electrons-rms is then related to a Ge-detector equivalent resolution by multiplying by $2.96 \mathrm{eV} / \mathrm{e}^{-}$and multiplying by 2.35 to obtain the FWHM resolution in eV. By fitting the individual noise components, one can attempt to isolate and mitigate the offending sources of noise. A minimum energy resolution of $224 \mathrm{eV}$ is observed for the large volume LBNL PPC detector as indicated in Fig. 4.

A smaller point contact detector fabricated at LBNL (Fig. 5) was mounted in a similar cryostat with a tensioned pin connecting the detector to the LMFE. The reduced point contact sizes provide a smaller capacitance $(<1 \mathrm{pF})$, and thus a better match to the low input capacitance of the JFET.

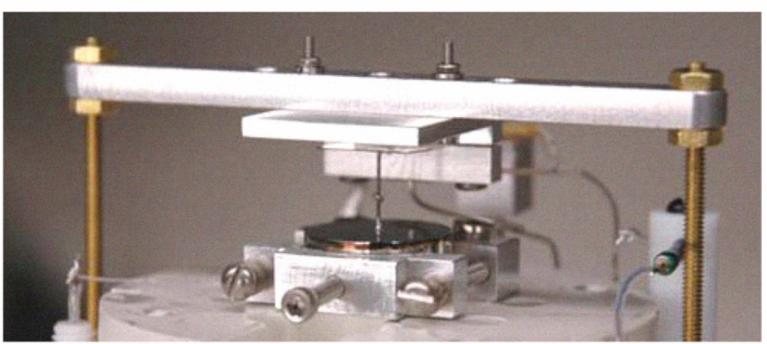

Fig. 5. Miniature PPC $(\varnothing 20 \mathrm{~mm} \times 10 \mathrm{~mm})$ mounted in a cryostat with copper pin connecting point contact to LMFE board, held (at one end) at $80 \mathrm{~K}$.

The noise performance of this Mini-PPC detector was measured in a similar fashion (Fig. 6).

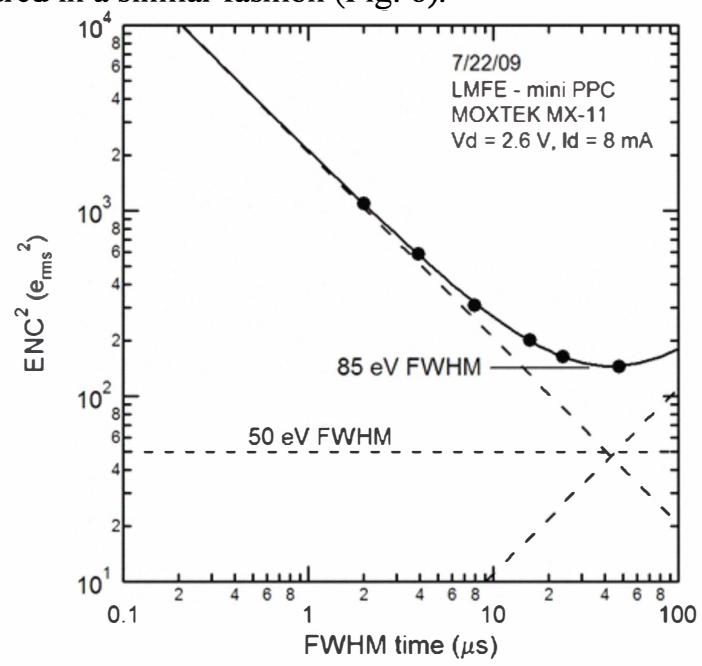

Fig. 6. Equivalent noise charge (squared) as a function of the FWHM equivalent shaping time for a Mini-PPC at drain-source voltage of $2.6 \mathrm{~V}$, and drain-source current of $8 \mathrm{~mA}$

A minimum noise level of $85 \mathrm{eV}$ FWHM was achieved, due in large part to the reduced detector capacitance. The minimum noise of the LMFE alone was measured at $55 \mathrm{eV} \mathrm{FWHM.}$

$\mathrm{An}^{241} \mathrm{Am}$ energy spectrum from this setup (Fig. 7) indicates an electronic noise of $95 \mathrm{eV}$ and a resolution of $378 \mathrm{eV}$ FWHM at $60 \mathrm{keV}$. This result is consistent with the $\mathrm{e}_{\mathrm{rms}}$ baseline noise measurement from Figure 6.

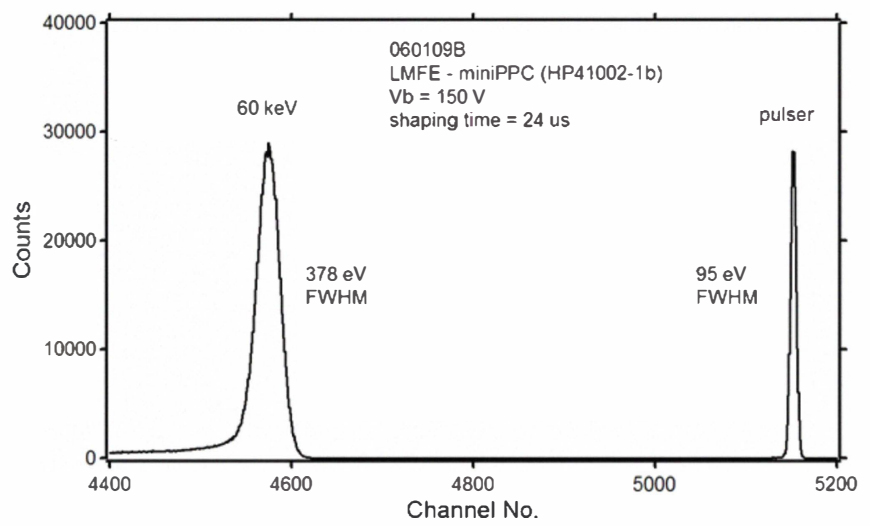

Fig. 7. Energy spectrum for ${ }^{241} \mathrm{Am}$ source incident on a Mini-PPC detector with LMFE readout. The low electronic noise of $95 \mathrm{eV}$ leads to a resolution of $378 \mathrm{eV}$ at $60 \mathrm{keV}$ for this system. 


\section{CONCLUSION}

Low noise levels made possible by low detector capacitance, low JFET capacitance and limited stray capacitances are critical for low energy or low threshold physics experiments. The requirement and availability of cooling to liquid nitrogen temperatures in germanium detector systems provides additional benefits to the performance of sensitive readout electronics. Although minimization of stray capacitance involves close proximity of the LMFE to the detector, the materials involved can be obtained from radiopure sources. Photolithography and hybrid circuit layout provide for a very low mass and low volume front end. It is expected that further optimization of this LMFE concept will make it an enabling technology for the next generation of low background physics experiments.

\section{REFERENCES}

[1] V.E. Guiseppe et. al., "The MAJORANA Neutrinoless Double-Beta Decay Experiment," 2008 IEEE Nucl. Sci. Symp., Conference Record, N22-1, 2008.

[2] C.E. Aalseth, P.S. Barbeau, D.G. Cerdeño et. al., "Experimental Constraints on a Dark Matter Origin for the DAMA Annual Modulation Effect," Phys Rev. Lett. 101, 251301, 2008.

[3] B. Cabrera-Palmer, D. Reyna, J. Steele, et. al., "Coherent Neutrino Nuclear Scattering With Germanium," presented at Applied Antineutrino Physics Workshop, Vienna, 2011.

[4] P.N. Luke, F.S. Goulding, N.W. Madden and R.H. Pehl, "Low Capacitance Large Volume Shaped-Field Germanium Detector," IEEE Trans. Nucl. Sci. vol. 36, no. 1, 1989.

[5] P.S. Barbeau, Neutrino and Astroparticle Physics with P-Type Point Contact High Purity Germanium Detectors. University of Chicago, Chicago, IL. PhD Dissertation, 2009.

[6] www.moxtek.com, Moxtek, Inc., Orem, Utah, USA 2011.

[7] P.W. Nicholson, Nuclear Electronics, Wiley, London 1974. 